\title{
Article \\ Photochemistry of 1-Phenyl-4-Allyl-Tetrazol-5-One: A Theoretical Study Contribution towards Mechanism Elucidation
}

\author{
Amilcar Duque-Prata (D), Carlos Serpa (D) and Pedro J. S. B. Caridade*(D) \\ Department of Chemistry, University of Coimbra, CQC Rua Larga, 3004-545 Coimbra, Portugal; \\ amilcar.prata@qui.uc.pt (A.D.-P.); serpasoa@uc.pt (C.S.) \\ * Correspondence: pedrojcaridade@uc.pt
}

Citation: Duque-Prata, A.; Serpa, C.; Caridade, P.J.S.B. Photochemistry of 1-Phenyl-4-Allyl-Tetrazol-5-One: A Theoretical Study Contribution towards Mechanism Elucidation. Appl. Sci. 2021, 11, 4045. https:// doi.org/10.3390/app11094045

Academic Editors: Benedetta Carlotti and David García-Fresnadillo

Received: 17 March 2021

Accepted: 27 April 2021

Published: 29 April 2021

Publisher's Note: MDPI stays neutral with regard to jurisdictional claims in published maps and institutional affiliations.

Copyright: (c) 2021 by the authors. Licensee MDPI, Basel, Switzerland. This article is an open access article distributed under the terms and conditions of the Creative Commons Attribution (CC BY) license (https:// creativecommons.org/licenses/by/ $4.0 /)$.

\begin{abstract}
The photodegradation mechanism of 1-phenyl-4-allyl-tetrazol-5-one has been studied using (time-dependent) density functional theory with the M06-HF, B3LYP, and PBE0 functionals and the VDZ basis set. All calculations have been carried out using the polarizable continuum model to simulate the solvent effects of methanol. The reaction pathway evolution on the triplet state has been characterised to validate a previously postulated experimental-based mechanism. The transition states and minimums have been initially located by local scanning in partial constrained optimisation, followed by a fully relaxed search procedure. The UV spectra has shown to be better described with PBE0 functional when compared with the experimental results, having the M06-HF a shift of $40 \mathrm{~nm}$. From the energetic point of view, the postulated mechanism has been validated in this work showing a concerted photoextrusion of the $\mathrm{N}_{2}$ molecule. The intramolecular proton transfer occurs at a later stage of the mechanism after cyclization of the allyl group on a triplet biradical intermediate. The photoproduct observed experimentally, a pyrimidinone, has been characterised. The infrared spectroscopic reaction profile has also been proposed.
\end{abstract}

Keywords: density functional theory; photochemistry; tetrazoles; reaction pathway; triplet state

\section{Introduction}

Tetrazoles are synthetic molecules containing a cyclic system with high aromaticity formed by four nitrogen atoms and one carbon atom [1,2]. Tetrazoles are being used in medicinal chemistry as bioisostere for carboxylic acids, as they originate from similar receptor-ligand interactions [3], as well as drugs in the treatment of several diseases, due the diversity of physical-chemical properties [4-7]. The energy materials industry also uses tetrazolic compounds as they are rich in nitrogen [8], have a high value of enthalpy of formation, thermal stability, explosive performance, and low sensitivity [9]. These compounds are used with different applications, from aerospace to the mining industry [10]. Tetrazoles are considered a more ecological variant since they produce $\mathrm{N}_{2}$ in combustion processes [11]. In chemical synthesis, they are widely used in the preparation of heterocyclic compounds with nitrogen atoms. Synthetic routes are described for various classes of compounds [12], using tetrazoles or derivatives, such as $9 H$-pyrimido(4,5-b)indoles [13], diaziridinones [14], iminoaziridines [15], carbodiimides [16], oxazines [17], benzimidazolones [16], and pyrimidinones [18-20].

Compounds with the tetrazol-5-one group (tetrazolone) are characterised by forming a carbonyl group with the ring carbon. The photochemistry of tetrazolic compounds focuses on ring cleavage, and the most frequently obtained products are azides, isocyanates, or aziridines. However, the presence of labile hydrogen atoms in the ring or substituting groups can lead to alternative reaction pathways, which occur simultaneously with other possible photochemical pathways. These processes can be favoured or hindered by the chemical nature and the conformational flexibility of the substituents linked to the tetrazolic ring, changing the relative quantities of various photoproducts [15,16,21-25]. 
Molecular nitrogen photoextrusion is common in tetrazolones photochemistry, and can occur with a concerted process, or through the formation of radical or zwitterionic species $[18,22]$. The photochemistry of tetrazolic compounds has been studied in solution and by matrix isolation. The matrix isolation approach limits the study to confined processes, eliminating alternative reaction pathways that could arise through molecular diffusion (except for small species produced), or that depend on conformational rearrangements $[18,22,23,26]$. Solution photoreactions have more associated variables, since it is known that the solvent and other reaction conditions have a direct influence on the yield and selectivity of the photodegradation of tetrazolic compounds [12].

The photochemistry of 1-phenyl-4-allyl-tetrazol-5-one (ATZ) has been studied with excitation at $254 \mathrm{~nm}$, and several reaction pathways have been proposed with the products characterised by various analytical techniques [17]. The photoproducts are strongly dependent of the solvent: Pyrimidinone was identified as the main product, in the case of protic solvents, and in aprotic solvents, isocyanates, and anilines. Based on the experimental observations, the photochemical mechanism of ATZ has been postulated for this reaction pathway, and it involves the formation of a triplet biradical intermediary after the photoextrusion of molecular nitrogen [18]. Pyrimidinone is formed after this intermediate undergoes a conformational rearrangement through the allylic group and a proton transfer. This proton transfer has been found to be intramolecular, as its occurrence is detected in both protic and aprotic solvents [18]. Based on the experimental detection of photoproducts, a mechanism has been postulated ad-hoc $[18,26]$, which assumes the relevance of the conformational rearrangements of the triplet intermediaries.

This work presents a theoretical analysis of the ATZ photodegradation mechanism focusing on the energetic properties of the reaction intermediates and photoproducts, considering a pathway which involves the formation of a ATZ triplet state before the molecular nitrogen photoextrusion. A detailed analysis of the mechanism may lead to a better understanding of the reactive pathways and create additional routes and selectivity in the chemical synthesis of ATZ. The relevant intermediates and transition states on the triplet potential energy surface has been characterised using density functional theory (DFT) and time-dependent DFT, employing the Tamm-Dancoff approximation [27], using the B3LYP [28,29], M06-HF [30], and PBE0 [31] functionals, together with Dunnings' cc-pVDZ basis set [32]. The leading states of the singlet electronic states have also been calculated, to interpret the UV-mechanics and to study the Frank-Condon vertical excitations. Considering the relevance of the solvent effects on the selectivity of the reaction, the conductor-like polarisable continuum model has been used $[33,34]$.

\section{Methods}

The photodegradation mechanism of ATZ in methanol has been suggested [18] taking into account the observed photoproduct pyrimidinone $(\mathrm{P})$. The mechanism, shown in Figure 1, involves the formation of a triplet biradical intermediary (M1), after the photoextrusion of molecular nitrogen. Pyrimidinone is formed after this intermediate undergoes a conformational rearrangement (M2) through the allyl group and an intramolecular proton transfer. Note that in the postulated mechanism, the pyrimidinone $(\mathrm{P})$ is in the singlet state, after an intersystem crossing from the triplet state, although not shown in Figure 1 and in Ref [18].

To validate the postulated mechanism, DFT and TDDFT electronic structure methods, as implemented in GAMESS [35], have been used to characterise energetically the relevant molecular intermediates and transition states. Methanol effects have been simulated by $\operatorname{PCM}[33,34]$ for all structures, in which it is described as a polarised dielectric medium and characterised by the macroscopic dielectric constant $\varepsilon=32.63$ obtained at $T=298 \mathrm{~K}$. A cavity of molecular shape hosts the solute, and is usually built as a series of interlocking spheres centred on the solute nuclei. The solvent polarisation is represented by an apparent surface charge spread on the cavity surface. In the computational implementation, the 
surface charge is discretised in finite elements and the PCM integral operators are replaced by their matrix representation [36].<smiles>C=CCn1nnn(-c2ccccc2)c1=O</smiles>

Figure 1. Postulated mechanism for the photodegradation of 1-phenyl-4-allyl-tetrazol-5-one (ATZ) in methanol [18].

Inherently to the (TD)DFT formulation is the issue related to the choice of the functional. Previously [19], B3LYP has been used to rationalise the vibrational spectroscopy of the ATZ in gas phase. Several studies $[37,38]$ have shown detailed benchmarks for a more reliable functional selection. It has been shown $[37,38]$ that M06-HF and PBE0 show better performance than B3LYP in a large set of systems, particularly in describing the barrier height of transition states and systems containing $\pi$ electrons. For the current work, the B3LYP functional was also employed, which provides a comparison with previous studies. Other possibilities could be also employed according to a process being studied as CAM-B3LYP [39] for vertical excitations, or B3LYP-D3 [40], LC-wPBE [41], wB97XD [42], and M06-2X [30] to account for long-rage forces. However, the selection of the functionals employed has been based on the benchmarks $[37,38]$ and as an accuracy compromise that may describe the complete photochemical reaction process here studied. To improve previous results, keeping a balance between accuracy and computational effort, the Dunning's cc-pVDZ basis-set [32] has been chosen for all calculations.

The molecular structures for the singlet ground-state, $S_{0}$, of ATZ and pyrimidinone have been optimised using DFT, and the RHF self-consistent field wavefunction. The triplet state of ATZ, pyrimidinone, and intermediates have been optimised using DFT with the ROHF self-consistent field wavefunction. Please note that the reaction mechanism occurs on the triplet state, except in the UV photoexcitation of ATZ and the final product. For the latter, both the singlet ground-state and the first triplet excited state has been characterised. The minimum localisation for all stable species has been carried out in a step-descent method using the quadratic approximation, with the gradient convergence criteria of $1 \times 10^{-4} \mathrm{E}_{\mathrm{h}} \mathrm{a}_{0}^{-1}$ for the largest component of the gradient and $1 / 3$ of that value for the root mean square gradient. After the stationary point geometry has been obtained, the harmonic vibrational calculation has been performed to confirm the minimum. For the transition states, the same criteria has been used but in a step-ascent approach, being confirmed by the presence of one imaginary frequency in the vibrational analysis.

\section{Results and Discussion}

\subsection{ATZ Singlet Electronic States}

ATZ shown in Figure 2 is known to have multiple conformers according to the spatial orientation of three main planes: Allylic group, tetrazolic, and phenyl rings. For the current work, the relevant conformers arise from the torsion of the allylic group, defined by the 
dihedral angle $\phi_{1-7-8-9}$. Figure 3 shows the dihedral angle dependence for partially optimised structures of ATZ- $S_{0}$ using PCM. For each point, an optimisation of all degrees of freedom, except the angle, is carried out to establish the torsion potential. The torsion behaviour of $S_{0}$ is similar for PBE0 and M06-HF functionals, the $\phi \sim 0$ deg being the most stable structure. For B3LYP, the most stable conformer is the one with $\phi=-124 \mathrm{deg}$; a similar trend has been also observed in gas-phase studies [19]. The energetic difference between the most stable conformer and the other minimums is larger in the case of M06HF $\left(2.6 \mathrm{~kJ} \mathrm{~mol}^{-1}\right)$. Note also that the M06-HF shows a more repulsive behaviour when departing from the global minimum, in particular for the \pm 180 deg with a barrier height of $11.5 \mathrm{~kJ} \mathrm{~mol}^{-1}$.

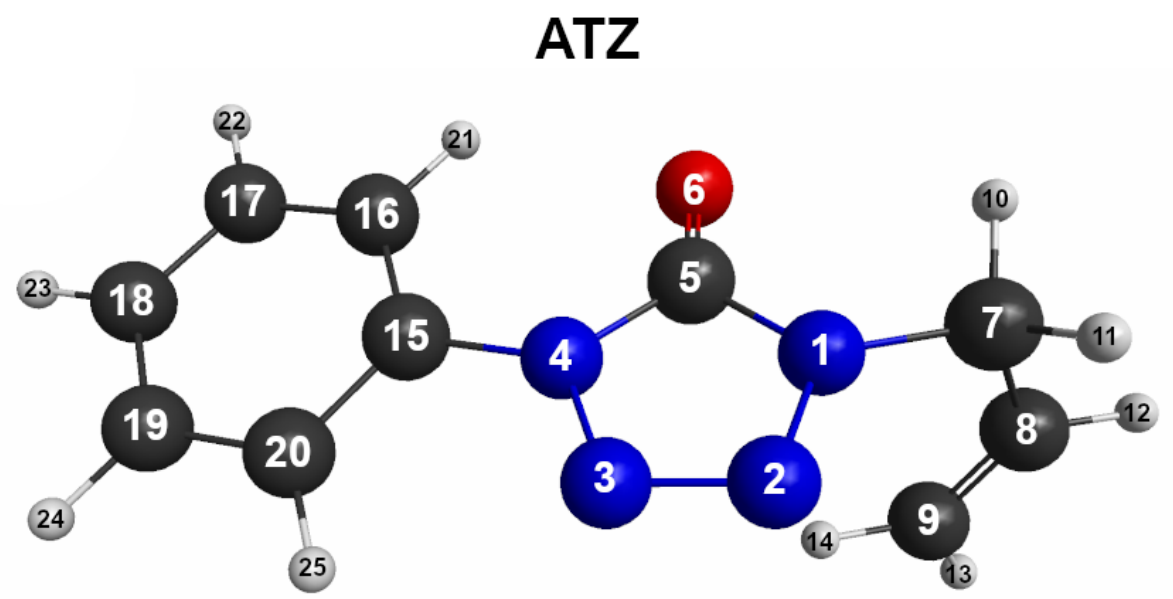

Figure 2. Singlet ground-state optimised ATZ molecule with the adopted atom labelling.

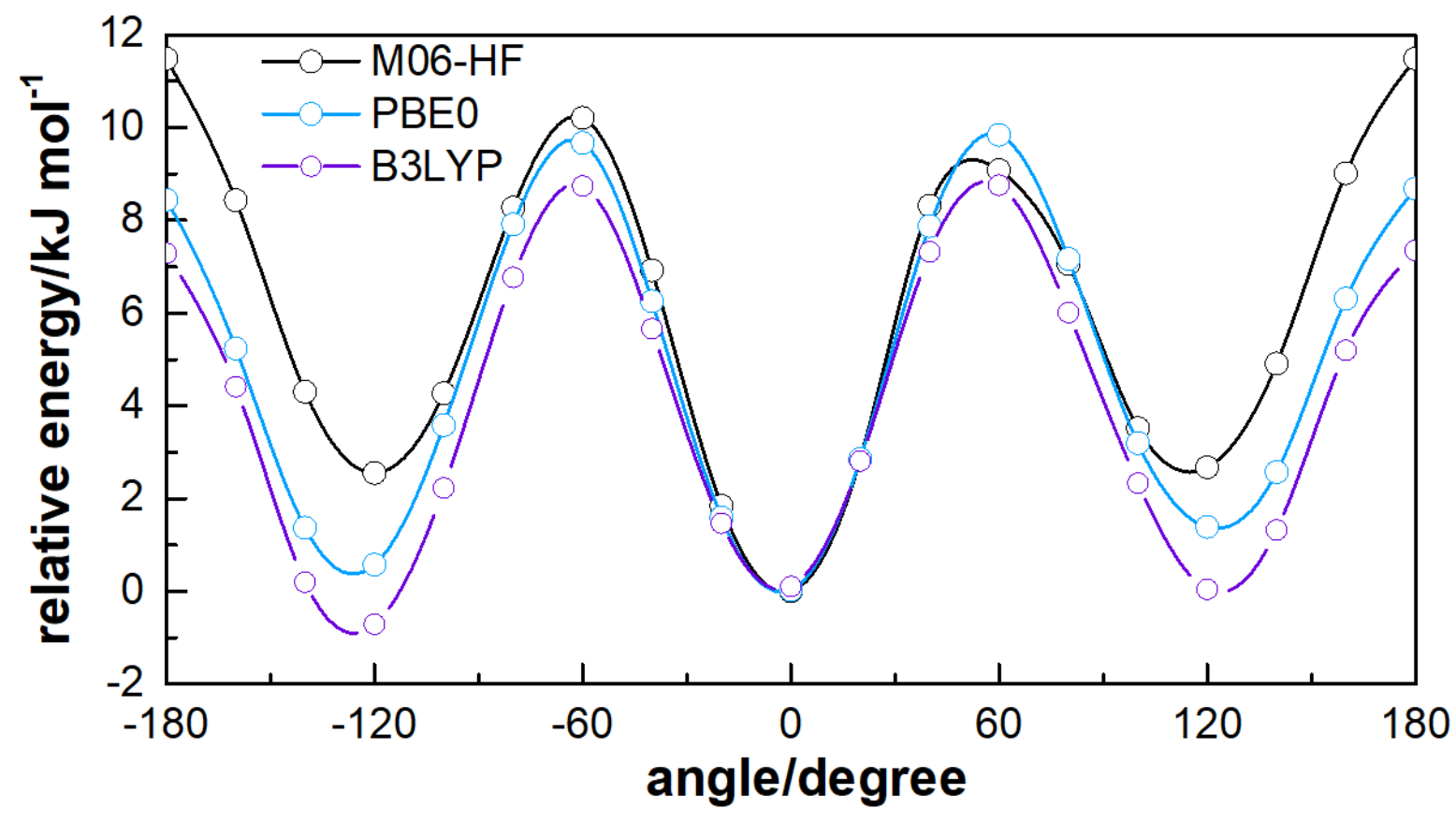

Figure 3. Partially optimised potential energy as a function of the conformational angle $\phi_{1-7-8-9}$. The lines are spline-type for a better analysis.

The relevant geometry parameters calculated for the ground-state $S_{0}$ with each functional are given on Table 1. For completeness, the full equilibrium geometry, energy, and spectroscopy properties are given in the Supplementary Materials. 
Table 1. Main structural parameters of optimised geometries of ATZ in the ground $\left(S_{0}\right)$ and triplet $\left(T_{1}\right)$ states using PCM. Distances are in Angstrom and angles in degrees. For a complete description of the molecule degrees of freedom see Supplementary Materials.

\begin{tabular}{|c|c|c|c|c|c|c|}
\hline \multirow[b]{2}{*}{ Property } & \multicolumn{2}{|c|}{ B3LYP } & \multicolumn{2}{|c|}{ M06-HF } & \multicolumn{2}{|c|}{ PBE0 } \\
\hline & $S_{0}$ & $T_{1}$ & $S_{0}$ & $T_{1}$ & $S_{0}$ & $T_{1}$ \\
\hline$r_{1-2}$ & 1.354 & 1.362 & 1.338 & 1.337 & 1.343 & 1.351 \\
\hline$r_{3-4}$ & 1.369 & 1.364 & 1.347 & 1.355 & 1.355 & 1.353 \\
\hline$r_{2-3}$ & 1.272 & 1.460 & 1.250 & 1.254 & 1.266 & 1.440 \\
\hline$r_{5-6}$ & 1.224 & 1.210 & 1.217 & 1.217 & 1.219 & 1.205 \\
\hline$r_{1-5}$ & 1.385 & 1.396 & 1.368 & 1.365 & 1.378 & 1.390 \\
\hline$r_{4-5}$ & 1.397 & 1.419 & 1.379 & 1.394 & 1.388 & 1.410 \\
\hline$r_{1-4}$ & 2.147 & 2.209 & 2.120 & 2.131 & 2.130 & 2.193 \\
\hline$r_{7-10}$ & 1.101 & 1.101 & 1.097 & 1.097 & 1.101 & 1.100 \\
\hline$\alpha_{1-2-3}$ & 108.7 & 103.3 & 108.9 & 109.1 & 108.7 & 103.2 \\
\hline$\alpha_{5-1-2}$ & 111.3 & 110.6 & 111.0 & 111.3 & 111.4 & 110.2 \\
\hline$\alpha_{5-4-3}$ & 110.2 & 109.9 & 110.2 & 109.5 & 110.5 & 109.6 \\
\hline$\alpha_{4-3-2}$ & 108.7 & 103.9 & 108.9 & 109.0 & 108.7 & 103.8 \\
\hline$\phi_{1-7-8-9}$ & -123.2 & 0.0 & 0.0 & -1.7 & -4.1 & 0.0 \\
\hline$\phi_{1-2-3-4}$ & 0.1 & 8.0 & 1.1 & 0.2 & 1.5 & 9.3 \\
\hline$\phi_{4-7-8-9}$ & -13.7 & -7.9 & -9.4 & -10.8 & -13.4 & -7.7 \\
\hline$\phi_{15-4-5-6}$ & 0.2 & 18.2 & -13.0 & -6.8 & -13.7 & 19.2 \\
\hline$\phi_{15-4-3-2}$ & 179.8 & -178.6 & 165.9 & 172.5 & 165.5 & -179.5 \\
\hline$\phi_{20-15-4-3}$ & 171.8 & -170.7 & 179.0 & 179.5 & 179.1 & -170.8 \\
\hline$\phi_{20-15-4-5}$ & 8.6 & -29.6 & 0.4 & 0.6 & 0.0 & -31.7 \\
\hline$E / E_{h}$ & -680.9182942 & -680.806160 & -681.161206 & -681.007779 & -680.550204 & -680.43570 \\
\hline$E_{0} / \mathrm{kJ} \mathrm{mol}^{-1}$ & 507.83 & 499.73 & 522.35 & 508.83 & 513.99 & 506.16 \\
\hline$v_{a} / \mathrm{m}^{-1}$ & 1772 & 1796 & 1810 & 1806 & 1824 & 1849 \\
\hline$v_{b} / \mathrm{cm}^{-1}$ & 1647 & 1640 & 1702 & 1694 & 1681 & 1673 \\
\hline$v_{c} / \mathrm{cm}^{-1}$ & 1524 & 1515 & 1565 & 1458 & 1544 & 1532 \\
\hline
\end{tabular}

It is clear that the studied functionals give the same results for the most relevant structural parameters, within the accuracy of the methods. Three of the most relevant normal modes for the reaction pathway of ATZ were also compared: $v_{a}$ correspond to the CO stretching, $v_{b}$ to the phenyl carbons stretching, and $v_{c}$ to the $\mathrm{N}(1)-\mathrm{C}(10)$ stretching. In addition, within the accuracy and limitations of the harmonic approximation, the larger zero-point energy $E_{0}$ can be found in the case of M06-HF and the lower value on the B3LYP. Such behaviour may be associated to the shape of the potential within the normal mode coordinates as observed in the literature [43].

The excitation transitions on the singlet states of ATZ at equilibrium geometry of the more stable conformer have been calculated using the TDDFT with the Tamm-Dancoff approximation [27]. Table 2 shows the vertical excitation energies, the components of the transition momentum, and the oscillator strength for the leading terms of the singlet manifold. In two of the functionals here studied, B3LYP and PBE0, the larger value of the oscillator strength is associated to the $S_{0} \rightarrow S_{1}$ transition $f \sim 0.385$, although the energy gap is $0.22 \mathrm{eV}$ larger in the case of PBE0. The M06-HF results show that the most intense transition corresponds to the $S_{0} \rightarrow S_{3}$ with a oscillator strength of 0.66 and an energy of $6.5 \mathrm{eV}$. From the TDDFT calculation, the absorption spectrum has been simulated, as shown in Figure 4, by using Gaussian functions centred at the calculated wavelengths and with bandwidths-at-half-height equal to $18 \mathrm{~nm}$.

The absorption spectrum in methanol has been measured with a Cary 5000 Series UV-Vis-NIR spectrophotometer (Agilent Technologies), with maximum absorption below 0.5 . Contrary to the gas phase spectrum, the possible absorption bands with wavelengths lower than $250 \mathrm{~nm}$ are masked by solvent absorption and are hence not shown in Figure 4. The experimental spectrum has (at least) two well defined shoulders at wavelengths higher than the main band maximum. Those are most probably due to the simultaneous changes in electronic and vibrational energy levels of the molecule (vibronic spectra). 
Vibronic spectra features are more notorious for molecules in both gas and apolar media, but (although more smooth and broadened) can also be seen in polar media. The band around $250 \mathrm{~nm}$ is due to the aromatic ring and the typical progression in the symmetric ring-breathing vibration with a value of about $1500 \mathrm{~cm}^{-1}$ observed, for example, see Serpa et al. [44]. As expected for a broadened spectra taken in polar media, the vibronic progression shoulders are more evident far from the main (high probability) absorption. The spectra that arises from the M06-HF shifts toward a lower wavelength centred at $200 \mathrm{~nm}$ although no experimental evidence corroborates such a behaviour. As expected from the oscillator strength and energy gap of the transitions, the B3LYP and PBE0 are centred in a region near the experimental value $\sim 250 \mathrm{~nm}$.

Table 2. Vertical excitation energies, transition dipole and oscillator strength for the leading terms of the singlet electronic manifold.

\begin{tabular}{cccccc}
\hline State & $\boldsymbol{\Delta E / \mathbf { e V }}$ & $\mu_{x} / \mathbf{D}$ & $\mu_{y} / \mathbf{D}$ & $\mu_{z} / \mathbf{D}$ & $f$ \\
\hline B3LYP & & & & & \\
$S_{1}$ & 4.828 & 1.7950 & 0.0207 & -0.1817 & 0.3850 \\
$S_{2}$ & 5.021 & -0.5511 & -0.0515 & 0.0439 & 0.0379 \\
$S_{3}$ & 5.266 & 0.0730 & -0.0003 & 0.2090 & 0.0063 \\
$S_{4}$ & 5.438 & -0.1353 & 0.0251 & -0.0600 & 0.0030 \\
$S_{5}$ & 5.670 & 0.6462 & -0.2856 & 0.0912 & 0.0705 \\
\hline M06-HF & & & & & \\
$S_{1}$ & 5.677 & 0.2548 & 0.0990 & -0.0488 & 0.0107 \\
$S_{2}$ & 6.003 & -0.0488 & 0.0136 & -0.3475 & 0.0181 \\
$S_{3}$ & 6.085 & 2.0851 & 0.1603 & -0.2364 & 0.6603 \\
$S_{4}$ & 6.544 & -0.2037 & -0.0297 & 0.1090 & 0.0087 \\
$S_{5}$ & 7.082 & -1.0043 & -0.1280 & 0.0216 & 0.1779 \\
\hline PBE0 & & & & & \\
$S_{1}$ & 5.042 & -1.7551 & -0.0594 & 0.1793 & 0.3849 \\
$S_{2}$ & 5.203 & -0.8622 & -0.0361 & 0.0711 & 0.0956 \\
$S_{3}$ & 5.499 & -0.1097 & -0.0062 & -0.2154 & 0.0079 \\
$S_{4}$ & 5.749 & 0.1360 & -0.0439 & 0.0636 & 0.0034 \\
$S_{5}$ & 5.962 & -0.5593 & 0.1908 & -0.1240 & 0.0533 \\
\hline
\end{tabular}

It is also important to note that there are considerable difficulties associated with the comparison of the computed vertical excitation energies with the experimental band maximum value. The experimental bands for all these systems are quite diffuse, meaning there can be considerable uncertainty in the positions of the band maximum. In addition, in the calculation of the vertical excitation energies, several effects that can significantly change the positions of the band maxima in the experimental spectrum were ignored. In particular, these calculations neglect the effects of vibrations (e.g., vibronic coupling and Jahn-Teller distortions) [45], spin-orbit coupling [46], and the environment [47], in cases where the spectra have been measured in solution or in a condensed medium. Hence, without including these additional effects in our calculations, there may be considerable discrepancies between the computed excitation energies and experimental band maxima, even with higher levels of theory and large basis sets. In general it has been observed that the computed vertical excitation energies are larger than the experimental band [48]. No attempt has been made to include the adiabatic and the 0-0 transitions in the absorption spectra [49]. 


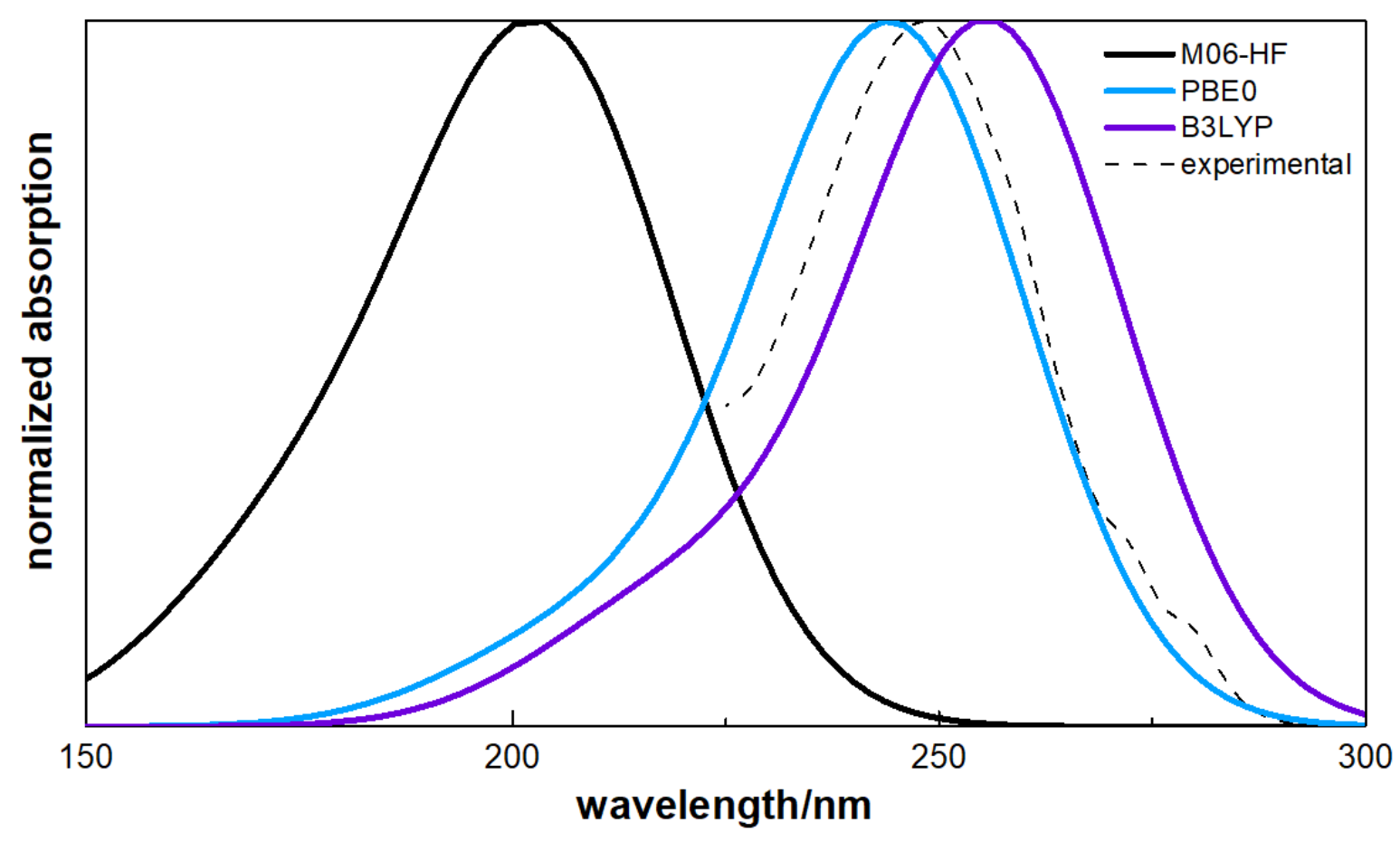

Figure 4. UV spectra for the ground-state of ATZ using the TDDFT/VDZ basis set with the Tamm-Dancoff approximation. For comparison the experimental result is also shown. Both experimental data and theoretical results have been obtain considering methanol as the solvent.

\subsection{Triplet Electronic States}

The proposed mechanism occurs from the triplet state of ATZ, populated from intersystem crossing with the singlet excited state. The geometry of the located global minimum for the lower triplet state, $T_{1}$, can be found in Table 1 for the three functionals (as before, the full coordinates may be found in the Supplementary Information). The minimum is located 293.4, 402.8, and $300 \mathrm{~kJ} \mathrm{~mol}^{-1}$ above the $S_{0}$ for the B3LYP, M06-HF, and PBE0, respectively. Such values may lead to an overestimation of the energy range for the case of M06-HF. However, such an analysis can only be tested with high-accuracy calculations which is out of the scope of the current work. Main changes of the $T_{1}$ state arises on the dihedral angles of the phenyl group except for the M06-HF functional.

Along the reaction pathway towards pyrimidinone, several transitions have been calculated. To obtain such structures, the z-matrix has been constructed to calculate a partial optimised profile in which all degrees of freedom are optimised except the critical angles and/or distances. For example, after populating the triplet electronic state, the system undergoes $\mathrm{N}_{2}$ photoextrusion in which the $\mathrm{N}(1)-\mathrm{N}(2)$ and $\mathrm{N}(3)-\mathrm{N}(4)$ bonds break. Those two variables have been fixed for different values until a critical point is obtained. This initial condition z-matrix is then used to obtain the real, non-constrained transition state. With this methodology, the calculated transition state connects the two minimum along the reaction path. The mechanism can be divided in three main steps, see Figure 5 for the stationary points on the reaction coordinate. Step 1 (a)-(b), denotes the two consecutive reactions that leads to the photoextrusion of $\mathrm{N}_{2}$ in ATZ, step 2 is the cyclization, and step 3 is the intramolecular proton transfer. 
(1a)

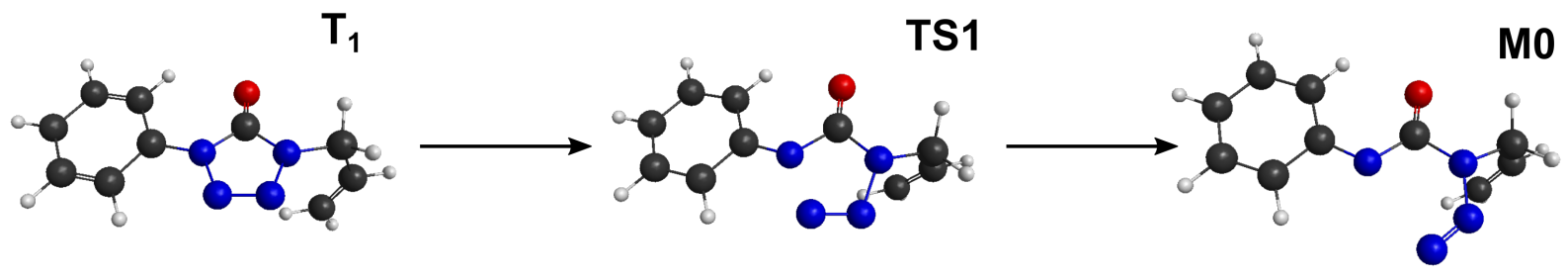

(1b)

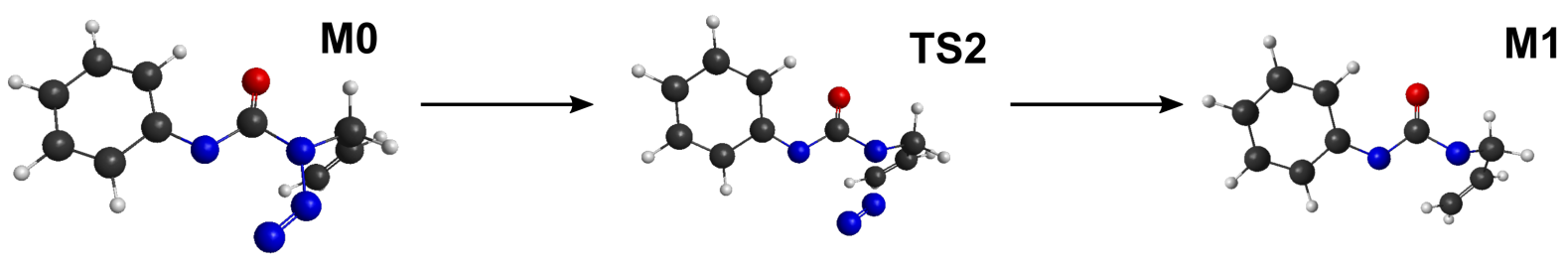

M1

(2)
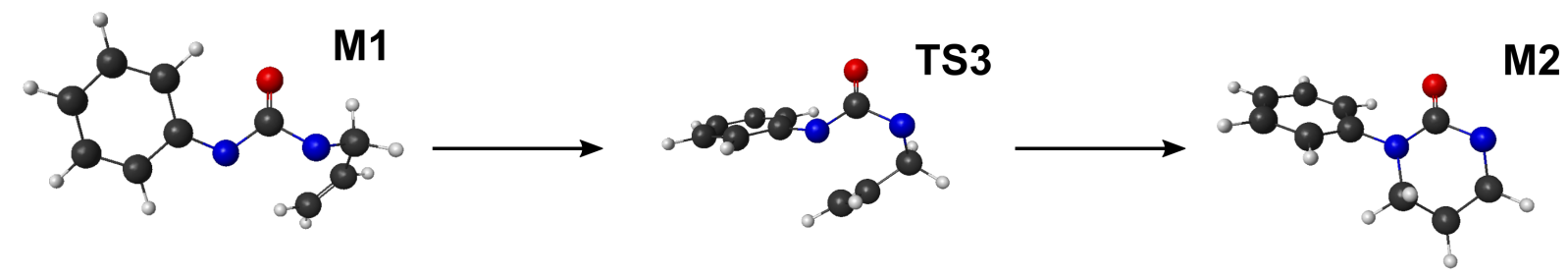

(3)
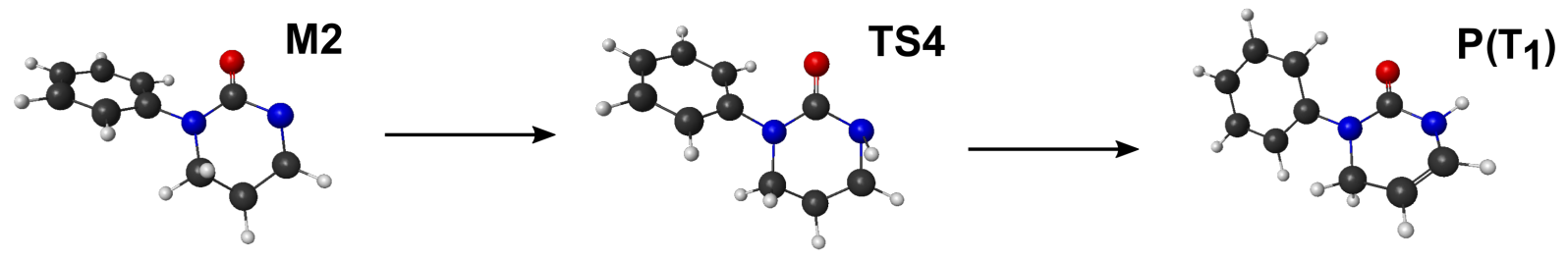

Figure 5. Structures along the pathway from $T_{1}$ towards pyrimidinone.

The photoextrusion of $\mathrm{N}_{2}$ in ATZ is still a subject of discussion [18]. It can occur through the triplet state or directly from a high-energy singlet excited state, and may occur via N-N simultaneous or concerted bond breaking channels. A scan of the partially relaxed pathways between the two channels has shown that the simultaneous bond-breaking is energetically unfavourable (by more than the double of the energy of TS1). Moreover, starting from several initial guess geometries in the simultaneous reaction coordinate, no transition-state has been found that connects $T_{1}$ to M1. In fact, the transition-state searches lead to the TS1 structure. In the non-concerted mechanism bond-breaking, two pathways have been studied: Starting with the rupture of $\mathrm{N}(1)-\mathrm{N}(2)$ or $\mathrm{N}(3)-\mathrm{N}(4)$ bonds. The energy barrier for the $\mathrm{N}(1)-\mathrm{N}(2)$ bond-breaking has been found to be $211 \mathrm{~kJ} \mathrm{~mol}^{-1}$ higher than the in the case of the $\mathrm{N}(3)-\mathrm{N}(4)$ bond-breaking. Therefore, the energy profile of the photodegradation has been studied considering the pathway that corresponds to the breaking of the $\mathrm{N}(3)-\mathrm{N}(4)$ bond in the first place, step 1a, followed by the breaking of the $\mathrm{N}(1)-\mathrm{N}(2)$ bond, step $1 \mathrm{~b}$. This $\mathrm{N}_{2}$ photoextrusion process can be translated as the passage from a geometry in which all the atoms in the two rings and the oxygen are in approximately the same plane, to one in which the different groups of the molecule are placed in three different planes: One plane for the phenyl, another for the NCON group, and another one for the allyic group.

The postulated mechanism states that M1 and possible M2 are biradicals intermediates. Open-shell biradicals can possess a multideterminant (multireference) character, which implies that single determinant methods will fail to describe these molecules in an appropriate way. Reliable descriptions can only be obtained with multireference results. For DFT, some approaches have also been proposed based on unrestricted methods [50]. 
In the current study, a simpler qualitative approach has been carried out by analysing the populations and free-valence that arise from the calculation. For the M1 intermediary, N(4) has a free valence value of 0.2 and $N(1)$ 0.6. These values show that such atoms will have a biradical character, in particular for N(1) atom. In the case of M2, the biradical character can be found located in atom $\mathrm{N}(4)$ (free valence value of 0.7 ) and $\mathrm{C}(8)$ (with 0.8 ). The birradical character may be also seen in the two semi-occupied molecular orbitals in Figure 6 as the M1 structure has a similar behaviour for all functions.

\section{M1}

M06-HF

B3LYP

PBE0
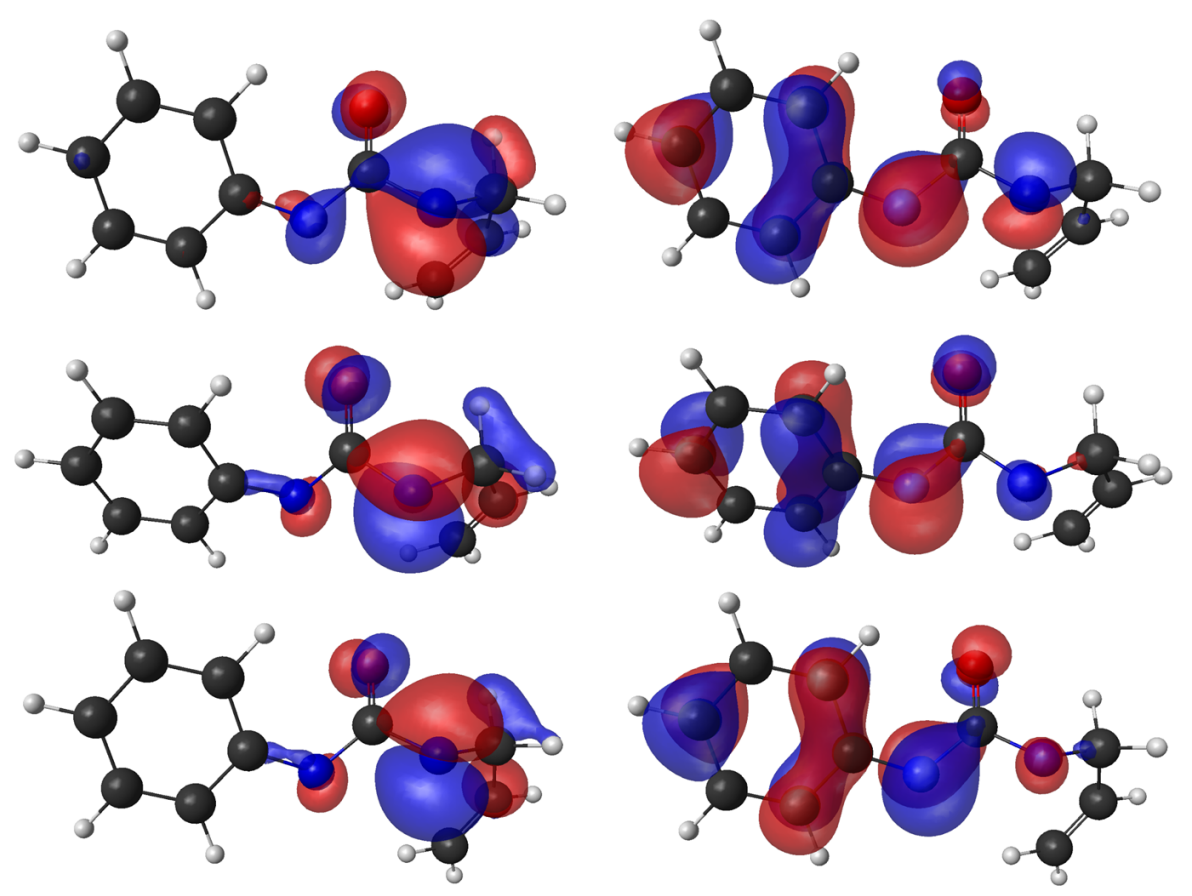

Figure 6. Semi-occupied orbitals of the M1 structure for the three functionals.

The transition states TS1 and TS2 have been located $354.5-458.9 \mathrm{~kJ} \mathrm{~mol}^{-1}$ and $223.2-$ $340.6 \mathrm{~kJ} \mathrm{~mol}^{-1}$ above $S_{0}$ according to the functional used. As previously shown, the B3LYP reveals the lower value and the M06-HF the higher, but considering the reaction path, $\Delta E^{\ddagger}=E_{\mathrm{TS} 1}-E_{T_{1}}$ and $\Delta E^{\ddagger}=E_{\mathrm{TS} 2}-E_{M 0}$, the TS1 in B3LYP has the larger barrierheight $\left(72.1 \mathrm{kcal} \mathrm{mol}^{-1}\right)$ and the lower relative energy barrier value $\left(3.8 \mathrm{kcal} \mathrm{mol}^{-1}\right)$ for the $\mathrm{M} 0 \rightarrow \mathrm{M} 1$ reaction. The vibrational analysis of the orthogonal normal mode relative to the reaction path is $398 i$ and $879 i$ for TS1 and TS2, respectively, associated to a stretching of the NN bond. The transition-state distances have been determined as $R_{12-13}=1.7214 \AA$ for TS1 and $R_{15-16}=1.61575 \AA$. The energy profile of this mechanism can be better seen on Figure 7.

After the extrusion of $\mathrm{N}_{2}$, the system undergoes a conformational rearrangement through the allylic group. The transition-state presents a barrier of $52.8 \mathrm{~kJ} \mathrm{~mol}^{-1}, 56.2 \mathrm{~kJ} \mathrm{~mol}^{-1}$, and $65.5 \mathrm{~kJ} \mathrm{~mol}^{-1}$, for M06HF, PBE0, and B3LYP, respectively. Although the barrier height is larger than the extrusion of $\mathrm{N}_{2}$, the shape of reaction coordinate at the critical point, associated to the imaginary frequency $471 \mathrm{icm}^{-1}$, shows a wider acceptance cone. Note, like steps $1 \mathrm{a}$ and $1 \mathrm{~b}$, the relative energy of the B3LYP and larger energy barrier suggests a more stable M2 species. 


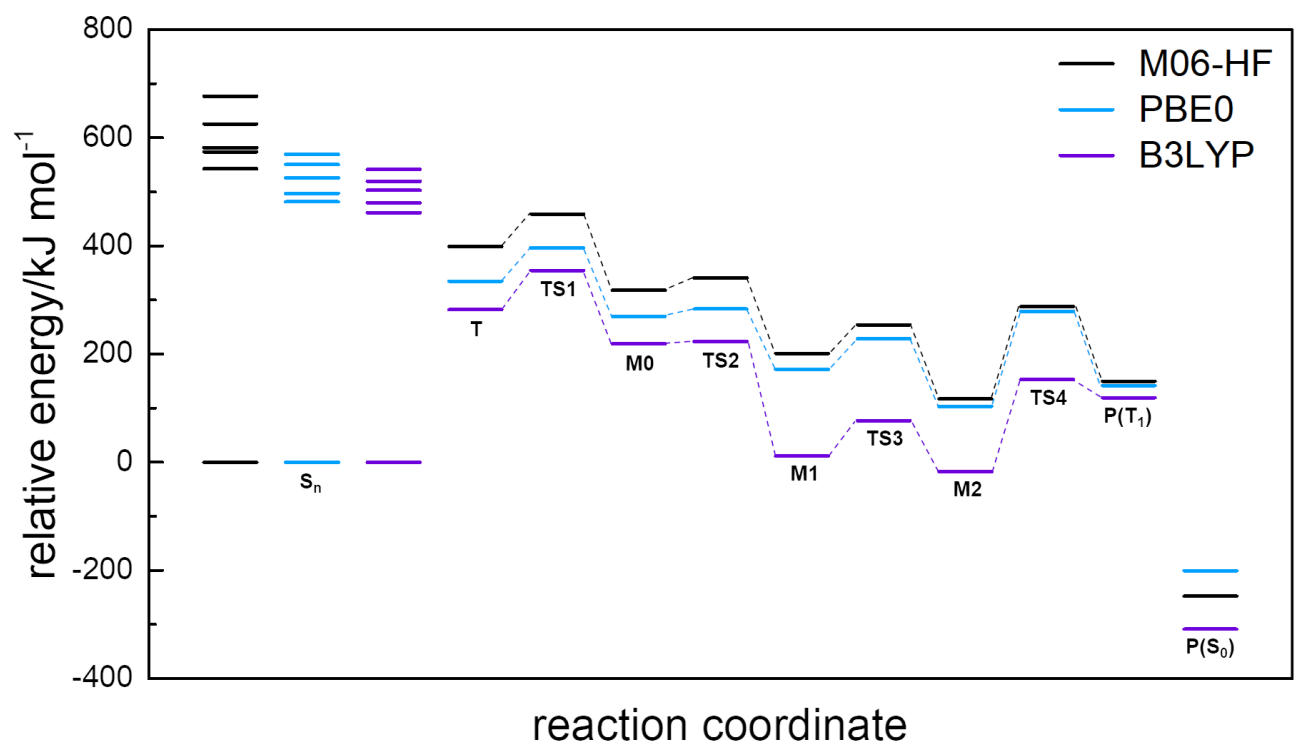

Figure 7. Relative energy profile for ATZ photoreaction, considering zero-point harmonic energy.

\subsection{Pyrimidinone}

The final pathway is related to the intramolecular proton transfer with the production of pyrimidinone. Several hypothesis may arise for this process, the most favourable process being the proton transfer (atom 10) from atom $\mathrm{C}(7)$ to $\mathrm{N}(1)$. The proton transfer is postulated to occur at the triplet state, and by intersystem crossing it goes to the singlet state. Both the triplet and singlet electronic state of pyridiminone have been studied to obtain the most stable structures. The minimum search in each potential energy surface follows the same method as before. Table 3 shows the relative energetic position of the singlet and triplet state of pyrimidinone.

Table 3. Energetic properties (in $\mathrm{kJ} \mathrm{mol}^{-1}$ ) of the relevant species along the reaction path: $\Delta E$, relative to the ground-state $\left(S_{0}\right)$ of ATZ; energy barrier $\Delta E^{\ddagger}=E^{\ddagger}-E_{\min }$ relative to the minimum prior to the transition-state.

\begin{tabular}{ccccccc}
\hline & \multicolumn{2}{c}{ M06-HF } & \multicolumn{2}{c}{ PBE0 } & \multicolumn{2}{c}{ B3LYP } \\
\cline { 2 - 6 } & $\boldsymbol{\Delta} \boldsymbol{E}$ & $\boldsymbol{\Delta} \boldsymbol{E}^{\ddagger}$ & $\boldsymbol{\Delta} \boldsymbol{E}$ & $\boldsymbol{\Delta} \boldsymbol{E}^{\ddagger}$ & $\boldsymbol{\Delta} \boldsymbol{\Delta}$ & $\boldsymbol{\Delta} \boldsymbol{E}^{\ddagger}$ \\
\hline$T_{1}$ & 398.9 & & 334.7 & & 282.4 & \\
TS1 & 458.9 & 60.0 & 396.2 & 61.5 & 354.5 & 72.1 \\
M0 & 318.4 & & 269.9 & & 219.3 & \\
TS2 & 340.6 & 22.3 & 283.3 & 13.4 & 223.2 & 3.8 \\
M1 & 201.1 & & 171.7 & & 11.5 & \\
TS3 & 254.0 & 52.8 & 228.0 & 56.2 & 77.0 & 65.5 \\
M2 & 117.0 & & 103.3 & & -17.3 & \\
TS4 & 287.9 & 170.9 & 279.2 & 175.9 & 152.9 & 170.2 \\
P(T $\left.T_{1}\right)$ & 149.5 & & 141.7 & & 119.4 & \\
P(S $\left.S_{0}\right)$ & -247.6 & & -201.0 & & -308.7 & \\
\hline
\end{tabular}

The transition-state, TS4, that connects M2 to the pyrimidinone triplet state has the larger energy barrier of the process $\left(\sim 170.2-175.9 \mathrm{~kJ} \mathrm{~mol}^{-1}\right)$, being the $\mathrm{M} 2 \rightarrow \mathrm{P}\left(\mathrm{T}_{1}\right)$ the limiting reaction process in the overall reaction. The imaginary frequency is $2211 \mathrm{icm}-1$ and is associated to the bending between $\mathrm{C}(7)-\mathrm{N}(1)-\mathrm{H}(10)$, in which the proton goes through the intramolecular migration. Pyrimidinone equilibrium geometry and energetics have been calculated showing that is more stable when calculated using the B3LYP and less stable with PBE0, a difference of nearly $100 \mathrm{~kJ} \mathrm{~mol}^{-1}$, and $50 \mathrm{~kJ} \mathrm{~mol}^{-1}$ for M06-HF. 


\subsection{Future Experimental Validation}

One of the main features of the present work is to validate the postulated mechanism for the photoreaction. From the energetic analysis of Figure 7, it is clear that the postulated mechanism is viable after population of excited singlet states $S_{n}$, as well from the calculated transition-states and reaction pathways. The analysis here presented may also be a key tool for the experimental validation, using, for example, time-resolved IR spectroscopy. In Figure 8 it is illustrated the normalised IR spectra calculated using a VDZ basis set and the three functionals.

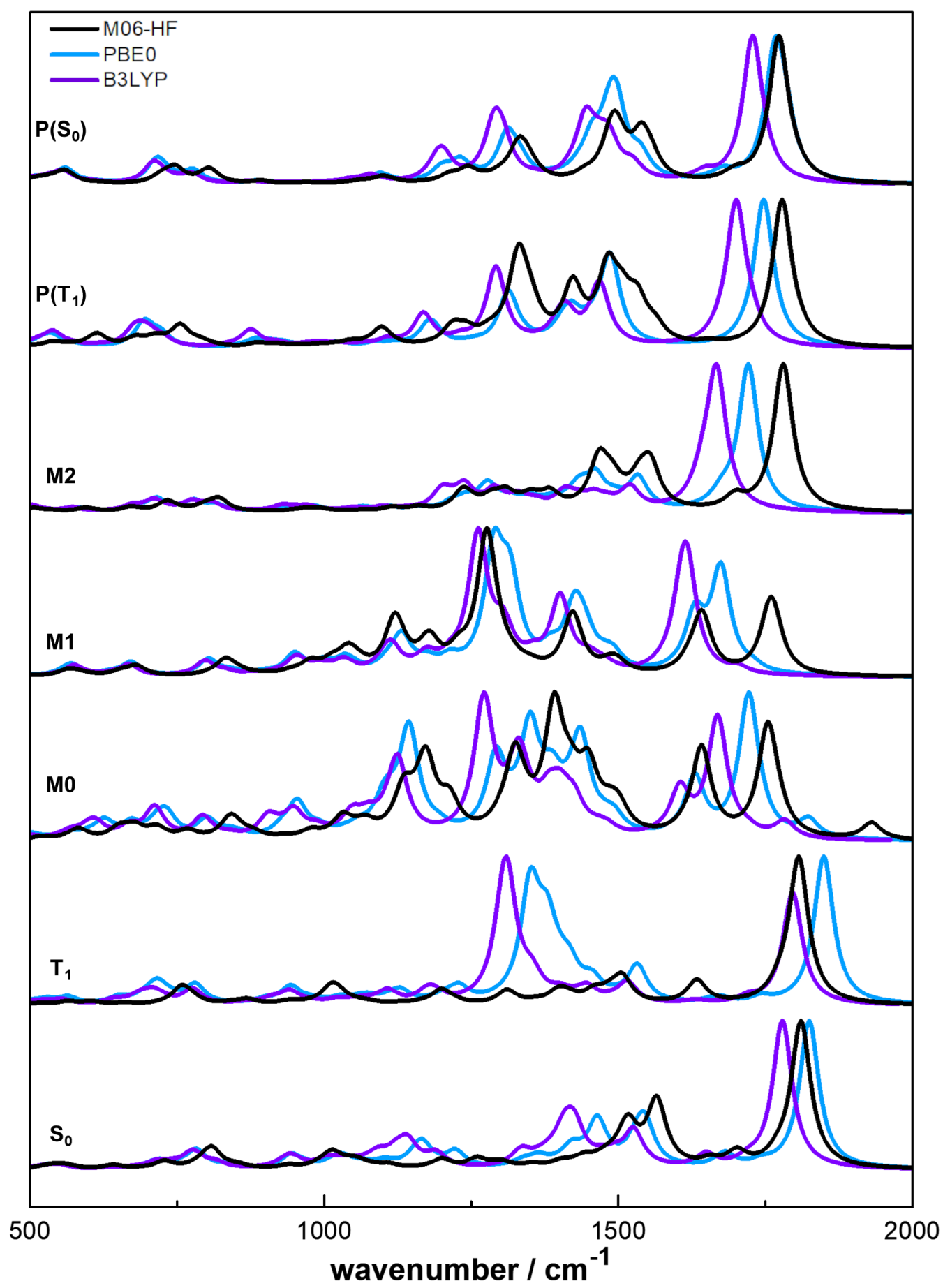

Figure 8. Normalised infrared spectra of the involved species in the photoreaction of ATZ. 
Analysing the reaction pathway, the change in the spectra of the two electronic states $S_{0}$ and $T_{1}$ is clear. In the ATZ triplet state spectrum the differences between M06-HF and the other two functionals are due to the different location of the semi-occupied orbitals above described: In the structure obtained with M06-HF, the semi-occupied orbitals are located in the phenyl group and in the obtained with PBE0 and B3LYP, and are located in the tetrazolic ring. The spectrum changes dramatically when the reaction undergoes the change towards M0. The new bands may be attributed to vibrational modes involving the $\mathrm{N}_{(4)}$ atom (around $1200 \mathrm{~cm}^{-1}$ ), the nitrogen atoms and the allylic group (around $1400 \mathrm{~cm}^{-1}$ ), and the nitrogen atoms and the phenyl ring (around $1700 \mathrm{~cm}^{-1}$ ). From the M1 to M2 spectra there are also some evident changes, since in the second, the band related to the stretching of the carbonyl group is significantly more intense than the others.

\section{Conclusions}

In this work, it has been shown that M06-HF, B3LYP and PBE0 functionals have a similar behaviour for the characterisation of ATZ and photoproducts. For the singlet state of ATZ, it has been shown that the more stable conformer occurs for the cis dihedral angle $\phi_{1-7-8-9}$ for M06-HF and PBE0, while B3LYP predicts a bend conformer with -124 deg. Major differences have been found in the singlet electronic states, with M06-HF predicting that the stronger transition may occur for $S_{0} \rightarrow S_{3}$. Such behaviour led to a shift in the simulated spectrum of about $40 \mathrm{~nm}$ to a lower wavelength. The remaining functionals described within $\sim 10 \mathrm{~cm}^{-1}$ the UV-Vis spectra of ATZ in methanol. Preliminary studies using equations of motion coupled-cluster theory with the 6-31G basis set, show also a good agreement with experimental spectra. Further studies for basis-set convergence are being carried out to access the quality of the functionals here employed. Other major effects may lead to changes of the overall spectra, since no non-adiabatic effects have been taken into account.

The proposed mechanism and pathways has been studied both energetically and structurally. In the description of the reaction profile, M06-HF and PBE0 lead to similar energy barriers values, as expected from the available literature. With B3LYP, the cyclization and the intramolecular proton transfer shows larger energy barriers and the species involved in these steps have lower energy values, a trend also observed in gas-phase studies. The marked changes between the calculated IR spectra of the various species allow to foresee that experimental validation of the mechanism through IR time-resolved spectroscopy is possible after this work, as long as adequate temporal resolution is available.

From the energetic point of view, the proposed mechanism [19] has been validated, although a more detailed analysis may require the use of molecular dynamical methods and multireference ab initio methods [51]. For the $S_{n}$ and $\mathrm{P}\left(T_{1}\right)$ states faith, the intersystem crossing, spin-orbit coupling, and conical intersection model will test the rate of conversion towards the $T_{1}$ state and competing processes, like fast decay to $S_{1}$, fluorescence, and phosphorescence. The use of an explicit solvation method [52] may also be relevant in the description of the interaction between the solvent and detail interactions with the solvent cage. Drift effects due to the solvent molecules may also change the steric behaviour of the rotations present in the mechanism.

Supplementary Materials: The following are available online at https:/ / www.mdpi.com/article/10.3 390/app11094045/s1: tables with the geometry, energy, and spectroscopy of the main relevant species.

Author Contributions: The work has been carried out by all authors in a equal basis. All authors have read and agreed to the published version of the manuscript.

Funding: This work has been financed by Portuguese funds through FCT—Fundação para a Ciência e a Tecnologia in the framework of the project UIDB/00313/2020.

Conflicts of Interest: The authors declare no conflict of interest. 


\section{References}

1. Myznikov, L.V.; Hrabalek, A.; Koldobskii, G.I. Drugs in the tetrazole series. Review. Chem. Heterocycl. Compd. 2007, 43, 1-9. [CrossRef]

2. Zhao, H.; Qu, Z.R.; Ye, H.Y.; Xiong, R.G. In situ hydrothermal synthesis of tetrazole coordination polymers with interesting physical properties. Chem. Soc. Rev. 2008, 37, 84-100. [CrossRef] [PubMed]

3. Zhao, T.; Kurpiewska, K.; Kalinowska-Tłuscik, J.; Herdtweck, E.; Dömling, A. $\alpha$-Amino Acid-Isosteric $\alpha$-Amino Tetrazoles. Chem. Eur. J. 2016, 22, 3009-3018. [CrossRef] [PubMed]

4. Rostom, S.A.F.; Ashour, H.M.A.; El Razik, H.A.A.; Abd El Fattah, H.; El-Din, N.N. Azole antimicrobial pharmacophore-based tetrazoles: Synthesis and biological evaluation as potential antimicrobial and anticonvulsant agents. Bioorganic Med. Chem. 2009, 17, 2410-2422. [CrossRef] [PubMed]

5. Bhaskar, V.H.; Mohite, P.B. Synthesis, characterization and evaluation of anticancer activity of some tetrazole derivatives. J. Optoelectron. Biomed. Mater. 2010, 2, 249-259.

6. Khanage, S.G.; Raju, A.; Mohite, P.B.; Pandhare, R.B. Analgesic activity of some 1,2,4-triazole heterocycles clubbed with pyrazole, tetrazole, isoxazole and pyrimidine. Adv. Pharm. Bull. 2013, 3, 13-18.

7. Trindade, N.R.; Lopes, P.R.; Naves, L.M.; Fajemiroye, J.O.; Alves, P.H.; Amaral, N.O.; Lião, L.M.; Rebelo, A.C.S.; Castro, C.H.; Braga, V.A.; et al. The Newly Synthesized Pyrazole Derivative 5-(1-(3 Fluorophenyl)-1H-Pyrazol-4-yl)-2H-Tetrazole Reduces Blood Pressure of Spontaneously Hypertensive Rats via NO-cGMO Pathway. Front. Physiol. 2018, 9, 1073. [CrossRef]

8. Ghule, V.D.; Radhakrishnan, S.; Jadhav, P.M. Computational studies on tetrazole derivatives as potential high energy materials. Struct. Chem. 2011, 22, 775-782. [CrossRef]

9. Shi, Y. Computational design of tetrazolone-based high-energy density energetic materials: Property prediction and decomposition mechanism. J. Phys. Org. Chem. 2018, 31, e3733. [CrossRef]

10. Fischer, D.; Klapötke, T.M.; Stierstorfer, J. 1, 5-Di (nitramino) Tetrazole: High Sensitivity and Superior Explosive Performance. Angew. Chem. Int. Ed. 2015, 54, 10299-10302. [CrossRef]

11. Klapötke, T.M.; Karaghiosoff, K.; Mayer, P.; Penger, A.; Welch, J.M. Synthesis and Characterization of 1,4-Dimethyl-5Aminotetrazolium 5-Nitrotetrazolate. Propellants Explos. Pyrotech. Int. J. Deal. Sci. Technol. Asp. Energ. Mater. 2006, 31, 188-195. [CrossRef]

12. Ismael, A.; Serpa, C.; Cristiano, M.L.S. Photochemistry of 1-allyl-4-aryltetrazolones in solution; Structural effects on photoproduct selectivity. Photochem. Photobiol. Sci. 2013, 12, 272-283. [CrossRef]

13. Hyatt, J.A.; Swenton, J.S. A facile synthesis of 9H-pyrimido [4,5-b] indole via photolysis of 4-azido-5-phenylpyrimidine. J. Heterocycl. Chem. 1972, 9, 409-410. [CrossRef]

14. Frija, L.M.T.; Ismael, A.; Cristiano, M.L.S. Photochemical transformations of tetrazole derivatives: Applications in organic synthesis. Molecules 2010, 15, 3757-3774. [CrossRef]

15. Quast, H.; Fuss, A.; Nüdling, W. Photoextrusion of Molecular Nitrogen from Annulated 5-Alkylidene-4, 5-dihydro-1H-tetrazoles: Annulated Iminoaziridines and the First Triplet Diazatrimethylenemethane. Eur. J. Org. Chem. 1998, 28, 317-327. [CrossRef]

16. Quast, H.; Nahr, U. Photochemische Stickstoff-Eliminierung aus 1, 4-Dihydro-1-phenyl-5H-tetrazol-5-onen und-thionen. Benzimidazolone und Carbodiimide. Chem. Berichte 1985, 118, 526-540. [CrossRef]

17. Frija, L.M.T.; Khmelinskii, I.V.; Serpa, C.; Reva, I.D.; Fausto, R.; Cristiano, M.L.S. Photochemistry of 5-allyloxy-tetrazoles: Steady-state and laser flash photolysis study. Org. Biomol. Chem. 2008, 6, 1046-1055. [CrossRef]

18. Frija, L.M.; Khmelinskii, I.V.; Cristiano, M.L.S. Mechanistic investigations into the photochemistry of 4-allyl-tetrazolones in solution: A new approach to the synthesis of 3,4-dihydro-pyrimidinones. J. Org. Chem. 2006, 71, 3583-3591. [CrossRef]

19. Frija, L.M.; Reva, I.D.; Gómez-Zavaglia, A.; Cristiano, M.L.; Fausto, R. UV-induced photochemistry of matrix-isolated 1-phenyl-4allyl-tetrazolone. Photochem. Photobiol. Sci. 2007, 6, 1170-1176. [CrossRef]

20. Dunkin, I.R.; Shields, C.J.; Quast, H. The photochemistry of 1,4-dihtdro-5h-tetrazole derivatives isolated in low-temperature matrices. Tetrahedron 1989, 45, 259-268. [CrossRef]

21. Quast, H.; Bieber, L. Synthese und Photolyse von 1, 4-Dialkyl-1, 4-dihydro-5H-tetrazol-5-onen und-thionen: Neue Wege zu Diaziridinonen und Carbodiimiden1. Chem. Berichte 1981, 114, 3253-3272. [CrossRef]

22. Gómez-Zavaglia, A.; Reva, I.D.; Frija, L.; Cristiano, M.L.; Fausto, R. Molecular structure, vibrational spectra and photochemistry of 2-methyl-2H-tetrazol-5-amine in solid argon. J. Phys. Chem. A 2005, 109, 7967-7976. [CrossRef]

23. Gómez-Zavaglia, A.; Reva, I.D.; Frija, L.; Cristiano, M.L.; Fausto, R. Photochemistry of 1-phenyl-tetrazolone isolated in solid argon. J. Photochem. Photobiol. A Chem. 2006, 179, 243-255. [CrossRef]

24. Ismael, A.; Cristiano, M.L.; Fausto, R.; Gómez-Zavaglia, A. Tautomer selective photochemistry in 1-(tetrazol-5-yl)ethanol. J. Phys. Chem. A 2010, 114, 13076-13085. [CrossRef]

25. Alawode, O.E.; Robinson, C.; Rayat, S. Clean photodecomposition of 1-methyl-4-phenyl-1 H-tetrazole-5 (4 H)-thiones to carbodiimides proceeds via a biradical. J. Org. Chem. 2011, 76, 216-222. [CrossRef]

26. Frija, L.M.; Khmelinskii, I.V.; Cristiano, M.L.S. Novel efficient synthesis of 3,4-dihydro-6-substituted-3-phenylpyrimidin-2(1H)ones. Tetrahedron Lett. 2005, 46, 6757-6760. [CrossRef]

27. Hirata, S.; Head-Gordon, M. Time-dependent density functional theory within the Tamm-Dancoff approximation. Chem. Phys. Lett. 1999, 314, 291-299. [CrossRef] 
28. Lee, C.; Yang, W.; Parr, R.G. Development of the Colle-Salvetti correlation-energy formula into a functional of the electron density. Phys. Rev. B 1988, 37, 785-789. [CrossRef]

29. Becke, A.D. Density-functional thermochemistry. III. The role of exact exchange. J. Chem. Phys. 1993, 98, 5648-5652. [CrossRef]

30. Zhao, Y.; Truhlar, D.G. The M06 suite of density functionals for main group thermochemistry, thermochemical kinetics, noncovalent interactions, excited states, and transition elements: Two new functionals and systematic testing of four M06-class functionals and 12 other function. Theor. Chem. Accounts 2008, 120, 215-241. [CrossRef]

31. Adamo, C.; Barone, V. Toward reliable density functional methods without adjustable parameters: The PBE0 model. J. Chem. Phys. 1999, 110, 6158-6170. [CrossRef]

32. Dunning Jr, T.H. Gaussian basis sets for use in correlated molecular calculations. I. The atoms boron through neon and hydrogen. J. Chem. Phys. 1989, 90, 1007-1023. [CrossRef]

33. Miertuš, S.; Scrocco, E.; Tomasi, J. Electrostatic interaction of a solute with a continuum. A direct utilizaion of AB initio molecular potentials for the prevision of solvent effects. Chem. Phys. 1981, 55, 117-129. [CrossRef]

34. Li, H.; Jensen, J.H. Improving the efficiency and convergence of geometry optimization with the polarizable continuum model: New energy gradients and molecular surface tessellation. J. Comput. Chem. 2004, 25, 1449-1462. [CrossRef]

35. Barca, G.M.; Bertoni, C.; Carrington, L.; Datta, D.; De Silva, N.; Deustua, J.E.; Fedorov, D.G.; Gour, J.R.; Gunina, A.O.; Guidez, E.; et al. Recent developments in the general atomic and molecular electronic structure system. J. Chem. Phys. 2020, 152, 154102. [CrossRef]

36. Cramer, C.J.; Truhlar, D.G. Implicit Solvation Models: Equilibria, Structure, Spectra, and Dynamics. Chem. Rev. 1999, 99, 2161-2200. [CrossRef]

37. Laurent, A.D.; Jacquemin, D. TD-DFT benchmarks: A review. Int. J. Quantum Chem. 2013, 113, 2019-2039. [CrossRef]

38. Coskun, D.; Jerome, S.V.; Friesner, R.A. Evaluation of the Performance of the B3LYP, PBE0, and M06 DFT Functionals, and DBLOC-Corrected Versions, in the Calculation of Redox Potentials and Spin Splittings for Transition Metal Containing Systems. J. Chem. Theory Comput. 2016, 12, 1121-1128. [CrossRef]

39. Yanai, T.; Tew, D.P.; Handy, N.C. A new hybrid exchange-correlation functional using the Coulomb-attenuating method (CAM-B3LYP). Chem. Phys. Lett. 2004, 393, 51-57. [CrossRef]

40. Grimme, S.; Antony, J.; Ehrlich, S.; Krieg, H. A consistent and accurate ab initio parametrization of density functional dispersion correction (DFT-D) for the 94 elements H-Pu. J. Chem. Phys. 2010, 132, 10089. [CrossRef]

41. Tawada, Y.; Tsuneda, T.; Yanagisawa, S.; Yanai, T.; Hirao, K. A long-range-corrected time-dependent density functional theory. J. Chem. Phys. 2004, 120, 8425-8433. [CrossRef]

42. Chai, J.D.; Head-Gordon, M. Long-range corrected hybrid density functionals with damped atom-atom dispersion corrections. Phys. Chem. Chem. Phys. 2008, 10, 6615-6620. [CrossRef]

43. Grimme, S.; Ehrlich, S.; Goerigk, L. Effect of the damping function in dispersion corrected density functional theory. J. Comput. Chem. 2011, 32, 1456-1465. [CrossRef]

44. Serpa, C.; Arnaut, L.G.; Formosinho, S.J.; Naqvi, K.R. Calculation of triplet-triplet energy transfer rates from emission and absorption spectra. The quenching of hemicarcerated triplet biacetyl by aromatic hydrocarbons. Photochem. Photobiol. Sci. 2003, 2, 616-623. [CrossRef]

45. Mckinlay, R.G.; Zurek, J.M.; Paterson, M.J. Vibronic Coupling in Inorganic Systems. Photochemistry, Conical Intersections, and the Jahn-Teller and Pseudo-Jahn-Teller Effects; Academic Press: Cambridge, MA, USA, 2010; Volume 62, pp. 351-390.

46. Rehmat, N.; Kurganskii, I.V.; Mahmood, Z.; Guan, Q.L.; Zhao, J.; Xing, Y.H.; Gurzadyan, G.G.; Fedin, M.V. Spin-Orbit ChargeTransfer Intersystem Crossing in Anthracene-Perylenebisimide Compact Electron Donor-Acceptor Dyads and Triads and Photochemical Dianion Formation. Chem. Eur. J. 2021. [CrossRef]

47. Cammi, R.; Corni, S.; Mennucci, B.; Tomasi, J. Electronic excitation energies of molecules in solution: State specific and linear response methods for nonequilibrium continuum solvation models. J. Chem. Phys. 2005, 122, 104513. [CrossRef]

48. Jacquemin, D.; Perpète, E.A.; Scuseria, G.E.; Ciofini, I.; Adamo, C. TD-DFT performance for the visible absorption spectra of organic dyes: Conventional versus long-range hybrids. J. Chem. Theory Comput. 2008, 4, 123-135. [CrossRef]

49. Fang, C.; Oruganti, B.; Durbeej, B. How method-dependent are calculated differences between vertical, adiabatic, and 0-0 excitation energies? J. Phys. Chem. A 2014, 118, 4157-4171. [CrossRef]

50. Gräfenstein, J.; Kraka, E.; Filatov, M.; Cremer, D. Can Unrestricted Density-Functional Theory Describe Open Shell Singlet Biradicals? Int. J. Mol. Sci. 2002, 3, 360-394. [CrossRef]

51. Coe, J.D.; Levine, B.G.; Martínez, T.J. Ab initio molecular dynamics of excited-state intramolecular proton transfer using multireference perturbation theory. J. Phys. Chem. A 2007, 111, 11302-11310. [CrossRef]

52. Raucci, U.; Perrella, F.; Donati, G.; Zoppi, M.; Petrone, A.; Rega, N. Ab-initio molecular dynamics and hybrid explicit-implicit solvation model for aqueous and nonaqueous solvents: GFP chromophore in water and methanol solution as case study. J. Comput. Chem. 2020, 41, 2228-2239. [CrossRef] 